\title{
A study on ESKAPE pathogens the BAD Bug with No Drug
}

\author{
K. Dinesh ${ }^{1}$, Mowna K.² \\ ${ }^{1}$ Dr. K. Dinesh, ${ }^{2}$ Dr. Mowna Karthick; both are Assistant Professor, Specialist Microbiology, Sree Balaji Medical \\ College and Hospital, Shri Sathya Sai Medical College and Research Hospital.
}

Address for Correspondence: Email: 01dineshdoc@gmail.com,

\begin{abstract}
ESKAPE pathogens include Enterococcus faecium, Staphylococcus aureus, Klebsiella pnuemonia, Acieneto bacter baumanii, Pseudomonas aeruginosa and Enterobacter species. Currently all these organisms are the main cause of hospital infections globally and they have the property to effectively escape the effect of antibacterial drugs. UNSTOPPABLE SUCCESS of these SUPERBUGS will lead to UNWINNABLE WAR. The success of these pathogens is mainly because of the mutations, modifications of LPS. As the crisis for the antibiotic resistance continues to grow, the latest IDSA (INFECTIOUS DISEASE SOCIETY OF AMERICA) "Bad Bugs, No Drugs"reports the urge for new antibiotics in the research and development pipeline and proposes steps to tackle the shortage. The aim of the study was to characterize the antimicrobial resistance in ESKAPE pathogens isolated from 330 culture positive clinical sample. Antibiotic resistance was determined by VITEK 2 and manual method was done on Kirby baurermethod. MIC was determined by VITEK 2 and E-Test according to CLSI guidelines. Out of the total cases 63 percent of the culture has ESKAPE pathogens. Except for S.aureus multidrug resistance index of ESKAPE pathogens revealed on increasing trend.in conclusion, ESKAPE pathogens are commonly identified in alarming frequency and knowledge of antimicrobial resistance will be aided for empirical treatment.
\end{abstract}

Keywords: ESKAPE pathogens, Multi drug resistance, infections, Antibiotics

\section{Introduction}

Nosocomial infections are caused by a variety of organisms, including bacteria, fungi, viruses, parasites, and other agents. Infections can be derived from exogenous or endogenous sources and are transferred by either direct or indirect contact between patients, healthcare workers, contaminated objects, visitors, or even various environmental sources. A survey of hospital-acquired infections (HAI) in the United States in 2011 reported a total of about 722,000 reported cases, with 75,000 deaths associated with nosocomial infections [8].

A second study conducted in 2002 estimated that when taking into account all types of bacterial infections, approximately 1.7 million patients suffered from HAIs, which contributed to the deaths of 99,000 patients per year [9]. From the last decade antibiotic resistance bacteria continue to be an challenge to the physicians. The growing numbers of antimicrobial-resistant pathogens, which are increasingly associated with

Manuscript received: $18^{\text {th }}$ June 2017

Reviewed: $28^{\text {th }}$ June 2017

Author Corrected; $8^{\text {th }}$ July 2017

Accepted for Publication: $16^{\text {th }}$ July 2017 nosocomial infection, place a significant burden on healthcare systems and have important global economic costs. There is steady increase in the curve of resistance development among the Gram Positive and Gram negative pathogens that cause infection in the hospital and community [1]. IDSA (Infectious Disease Society of America) reported these as the ESKAPE pathogens Enterococcus faecium, Staphylococcus aureus, Klebsiella pneumonia, Acinetobacter baumanii, Pseudomonas aeruginosa, Enterobacter species) these are responsible for the majority of hospital acquired infections and they also effectively "ESCAPE" the effects of antibacterial drugs.

Data from the centre for disease control and preventions show a rapid increase in the rate of infection due to Methicillin- resistant S.aureus (MRSA), Vancomycinresistant E. faecium, and fluoroquinolone-resistant P.aeruginosa [4]. There has been a increase in reporting of infections caused by Multi Drug resistant organisms, thereby limiting the choice of effective antimicrobial agents available to clinicians. The addition of aging 


\section{Original Research Article}

population and frequent referral of patients to acute care facilities also add for the prevalence of multi drug resistant organisms. Multidrug resistant (MDR) organisms especially gram negative bacilli have become a pivotal of long term care facilities in the hospital and vice-versa [5]. In contemporaneous the aging population and frequent referrals of patients from and to acute care facilities also add as the reservoir for the MDR [1]. The unstoppable success of these SUPERBUGS will lead to the crisis called "UNWINNABLE WAR" [6]. Data from the National Nosocomial Infection Surveillance (NNIS) System (2003 versus 1998- 2002) showed that, in the nine selected antimicrobial-resistant pathogens associated with nosocomial infections in intensive care unit patients, there is increase in the prevalence of resistance to third-generation cephalosporins (either ceftriaxone, cefotaxime or ceftazidime.

The emergence of resistant organisms to other drugs serves to bring the therapeutic importance of polymyxins such as colistin. No new antibiotic classes against multi drug resistant Gram-negative bacteria are expected to be commercially available within the next several years. Even more worrying, the emergence of resistance to colistin, the only available active antibiotic against multidrug-resistant Gram-negative bacteria [7].

Our therapeutic options for these pathogens are so extremely limited that clinicians are forced to use older, previously discarded drugs, such as colistin, that are associated with significant toxicity and for which there is a lack of robust data to guide selection of dosage regimen or duration of therapy [8]. The growing number of elderly patients and patients undergoing surgery, transplantation, and chemotherapy and dramatic increases in population in neonatal intensive care units will produce an even greater number of immunocompromised individuals at risk of these infections [9].

Antimicrobial resistance pattern of ESKAPE
Pathogens- Antimicrobial resistance genes may be
carried on the bacterial chromosome, plasmid, or
transposons [10]. Mechanisms of drug resistance fall
into several broad categories, including drug

\section{Results}

In total 1000 samples, 430 culture positive clinical sample. Among 430 Positive samples, 271 pathogens were identified as ESKAPE pathogens. The Enterococcus faecium accounted for 28, 72 samples isolated MRSA. In 64 and 39 samples Pseudomonas aeruginosa and acinetobacter were isolated respectively. 46 samples isolated Klebsiella pneumonia and 22 isoated Enterobacter. inactivation/alteration, modification of drug binding sites/targets, changes in cell permeability resulting in reduced intracellular drug accumulation, and biofilm formation [11-12].

- Drug Inactivation or Alteration

- Modification of Drug Binding Sites

- Reduced Intracellular Drug Accumulation

- Biofilm Formation

\section{Methodology}

The research done is a applied, analytical type of case study research. The samples where collected by direct observational method. All the ESKAPE PATHOGENS isolated samples where included in the study (probability sampling).

To avoid multiple entries from a single patient, only the first positive MDR culture for a given patient was included. The patients Identification number, age, sex, type of sample, recent significant treatment history with antibiotics, provisional diagnosis, duration of hospital stay and any other history related to the research was collected in observational design from the administrative data base. All the clinical samples received for Bacteriological culture in Microbiology section of the laboratory were processed and analyzed for the research. All clinical samples were inoculated in the respective media and methods as per standard guidelines and incubated.The blood culture bottles will be placed in Bac T/ Alert $3 \mathrm{D}$ and the positive culture bacteriological media for inoculation and incubated. All the ESKAPE pathogens isolated from all the clinical samples will be subjected for determining the MIC and Sensitivity by Vitek 2 and Kirby Bauer method as per CLSI.

Isolates that were collected within 2 days after admission were considered to be acquired prior to the hospitalization, or "nonnosocomial"; Isolates acquired after day 2 were considered "nosocomial." Total of 1000 samples were included in the study after the ethical committee approval by the institution. bottle will be processed by Grams stain and in routine 
Original Research Article

The samples which were resistant to more than 3 groups of antibiotic were considered as Multidrug Resistant Organisms (MDRO). Out of 1000 samples $6.9 \%$ were MDR.

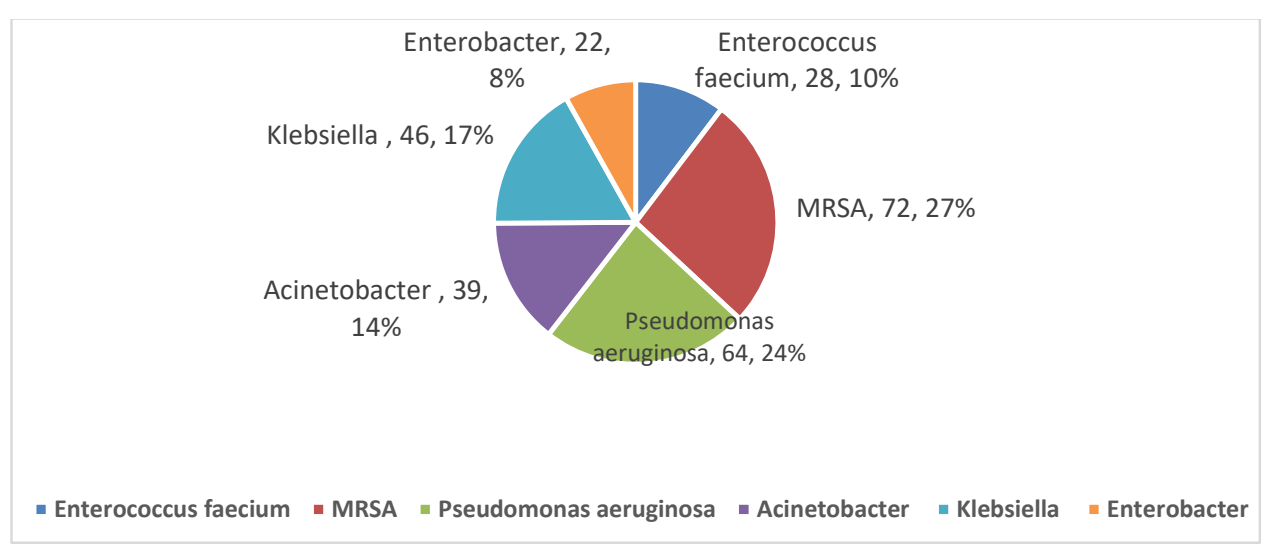

Figure-1: Distribution of ESKAPE

\section{Discussion}

The bacteria has the ability to rapidly gain resistance along with overuse and misuse of antibiotics, we are now living in the age of multidrug resistant (MDR) and Pan-drug resistant (PDR) bacterial pathogens leading to the situation like the pre-antibiotic era. This is due to the improper use of antibiotics and the free availability of antibiotics to public. We live in a period where even though people are well educated they refuse to consult a Doctor for the disease, and purchase the antibiotics from the pharmacy counter freely without a valid physicians prescription. One more consent for the development of MDR bacteria is that most of the reserved antibiotics have now come to the market for day today use and now we don't have a reserve antibiotics to take care of the resistant bugs. Most of the broad spectrum antibiotics have now come in simple tablet and injection form adding to vowe of MDR bacteria. The patients too donot take the antibiotics as prescribed by the physicians and they discountinue there course leading to the development of MRD.

The increased burden of antimicrobial resistance has been due to the increased days of stay in hospital, mortality, and the cost of Hospital care (10). Of more recent concern is the emergence of MDR Gramnegative bacilli (GNB) in long term care facilities; several studies have shown that the carriage prevalence of MDR GNB has far exceeded that of methicillinresistant Staphylococcus aureus (MRSA) and vancomycin- resistant enterococci (VRE)(4). Acineto bacter baumannii, Pseudomonas and Enterobacteriaceae were considered to be Pan drug resistant (PDR) if isolates were resistant to all classes of anti pseudomonalagents $(11,12)$. This study focuses on the resistance pattern of the most commonly isolated MDR organism and emergence of antimicrobial stewardship to be followed by the physicians.

\section{Conclusion}

The study mainly concerns of resistance pattern in the ESKAPE pathogens which is the major threat to hospital as well as community acquired infections. In our study the most common pathogen was found to be MRSA which is frequently seen in the hospitals, it is the duty of the treating physician and health works to use proper hand hygiene techniquewhich can minimize the risk of spread of MRSA to the community. In our study Pseudomonas aeruginosa was the second most common pathogen showing a wide range of resistance pattern, and in particular they where resistant to Imipenem which is an alarming issue. There should be a strict antibiotic stewardship to be followed in each hospitals and restricted use of antibiotics as per sensitivity pattern must be made mandatory in all the hospitals and clinics. And the use of antibiotics including the dosage of each drugs, number of days the drug is prescribed should be monitored periodically. Sale of antibiotics must be monitored and drugs without a valid prescription should not be encouraged. Patients should also be monitored and should be encouraged to take the full course of antibiotics and not to discontinue the medications. All these measure will ensure to reduce the number of MDR bugs in our environment.

Acknowledgements: I would like to express my gratitude to my professor Dr. Venugopal, for helping me with this study. 


\section{Original Research Article}

\section{Funding: Nil, Conflict of interest: None.} Permission of IRB: Yes

\section{References}

1. Giske CG, Monnet DL, Cars O, Carmeli Y; ReActAction on Antibiotic Resistance.Clinical and economic impact of common multidrug-resistant gram-negative bacilli. Antimicrob Agents Chemother. 2008 Mar; 52

(3): 813-21. Epub 2007 Dec 10.

2. Rice LB. Federal funding for the study of antimicrobialresistance in nosocomial- pathogens: no ESKAPE.J Infect Dis. 2008 Apr 15;197(8):1079-81. doi: 10. 1086/ 533452 .

3. Spellberg B, Guidos R, Gilbert D, Bradley J, Boucher HW, Scheld WM, Bartlett JG, Edwards J Jr; Infectious Diseases Society of America. The epidemic of antibiotic-resistant infections: a call to action for the medical community from the Infectious Diseases Society of America. Clin Infect Dis. 2008 Jan 15;46(2): 155-64. doi: 10.1086/524891.

4. National Nosocomial Infections Surveillance System Report, data summary from January 1992 through June (2004) issued October 2004. Am J Infect Control 2004; $32: 470-85$.

5. Lim CJ, Cheng AC, Kennon J, Spelman D, Hale D, Melican G, Sidjabat HE, Paterson DL, Kong DC, Peleg AY. Prevalence of multidrug-resistant organisms and risk factors for carriage in long-term care facilities: a nested case-control study. J Antimicrob Chemother. 2014 Jul;69(7):1972-80. doi: 10.1093/jac/dku077. Epub 2014 Apr 7.

6. Federico Perez, Andrea M. Hujer, Kristine M. Hujer, Brooke K. Decker, Philip N. Rather, and Robert A.
Bonomo. Global Challenge of Multidrug-Resistant Acinetobacterbaumannii ANTIMICROBIAL AGENTS AND CHEMOTHERAPY, Oct. (2007), p. 3471-3484 Vol. 51, No. 10 0066-4804/07/\$08.000 doi:10. 1128 /AAC. 01464-06 Copyright (C) 2007, American Society for Microbiology.

7. Anima Poudyal, Benjamin P. Howden In vitro pharmacodynamics of colistin against multidrugresistant Klebsiella pneumonia new Journal of Antimicrobial Chemotherapy (2008) 62, 1311-1318 doi: 10. 1093/jac/dkn425 Advance Access publication 15 October (2008)

8. Magill S. S., Edwards J. R., Bamberg W., et al. Multistate point-prevalence survey of health careassociated infections. The New England Journal of Medicine. 2014; 370 (13):1198-1208. doi: 10.1056/ nejmoa1306801. [PMC free article] [PubMed] [Cross Ref]

9. Klevens R. M., Edwards J. R., Richards C. L., Jr., et al. Estimating health care-associated infections and deaths in U.S. Hospitals, 2002. Public Health Reports. 2007; 122(2):160-166. [PMC free article] [PubMed]

10. Giedraitienè A, Vitkauskienè A, Naginienè R, Pavilonis A. Antibiotic resistance mechanisms of clinically important bacteria. Medicina (Kaunas). 2011; 47(3):137-46.

11. Wright G. D. Bacterial resistance to antibiotics: enzymatic degradation and modification. Advanced Drug Delivery Reviews. 2005;57(10):1451-1470. doi: 10.1016/j.addr.2005.04.002. [PubMed] [Cross Ref]

12. Wilson DN. Ribosome-targeting antibiotics and mechanisms of bacterialresistance. Nat Rev Microbiol. 2014 Jan;12(1):35-48. doi: 10.1038/nrmicro3155.

\section{How to cite this article?}

K. Dinesh, Mowna K. A study on ESKAPE pathogens the BAD Bug with No Drug. Trop J Ophthalmol Otolaryngol.2017;2(2):19-22. doi: 10.17511/jooo.2017.i02.01. 